Z. klin. Chem. u. klin. Biochem.

7. Jg., S. $581-585$, November 1969

\title{
Die Jaffé-Reaktion mit Kreatinin Reaktionsprodukt und allgemeine Reaktionsbedingungen
}

\author{
Von H. P. SeELIG \\ Aus dem Med.-Diagnostischen Institut der Städt. Krankenanstalten Karlsrube (Chefarzt: Dr. H. Wüst)
}

(Eingegangen am 9. Juni 1969)

Spektroskopische und chromatographische Untersuchungen der Jaffé-Reaktion mit Kreatinin ergeben, daß das rote Reaktionsprodukt nicht mit der häufig dafür angegebenen Pikraminsäure identisch ist. Es scheint vielmehr ein Additionskomplex aus einem Molekül Kreatinin und einem Molekül Pikrinsäure zu sein, der nur in alkalischer Lösung stabil ist.

Hohe Alkalität unterdrückt die Bildung des roten Farbstoffs aus Kreatinin, jedoch wird roter Farbstoff durch $\mathrm{NaOH}$ selbst gebildet, der spektroskopisch und visuell nicht von dem aus Kreatinin gebildeten zu untcrscheiden ist. Eine geringere Farbausbeute wurde auch mit hoher Konzentration von Kreatinin gefunden. Das kann durch Bildung einer Verbindung aus zwei Molekülen Kreatinin und einem Molekül Pikrinsäure bedingt sein.

Für klinische Zwecke müssen zu hohe $\mathrm{NaOH}-$ Konzentration und $\mathrm{NaOH}-$ Konzentrationsdifferenzen in Standard, Leerwert und Probe vermieden werden. Die photometrische Messung sollte bei $\lambda>490 \mathrm{~nm}$ ausgeführt werden, um eine bessere Linearität zivischen Kreatininkonzentration und Extinktion zu crhalten.

\section{The Jaffé reaction with creatinine. The reaction product and general reaction conditions}

Spectroscopic and chromatographic studies of the Jaffé-reaction with creatinine revealed that the red product of the reaction has no identity with the frequently suggested picramic acid. It is thought to be an addition complex of one molecule of creatinine and one molecule of picric acid, which may only be stable in alkaline solution.

High alkalinity suppresses formation of the red colour from creatinine, but a red colour is produced by the $\mathrm{NaOH}$ itself, which is spectroscopically and visually indistinguishable from that produced by creatinine. Loss of colour was also found with high concentrations of creatinine. This may be due to the formation of a di-creatinine picric acid compound.

For clinical purpose, high $\mathrm{NaOH}$ concentrations and variations of the $\mathrm{NaOH}$ level within standard, blank, and test solution should be avoided. The photometric measurement should be performed at wavelengths greater than $490 \mathrm{~nm}$, which give an improved linearity between creatinine concentration and extinction.

Zwei Drittel der zahlreichen zur Kreatininbestimmung entwickelten Methoden beruhen auf der Jaffé-Reaktion (1): Alkalische Pikrinsäurelösung zeigt kurze Zeit nach Kreatininzugabe eine Rotfärbung, deren Absorptionsintensität sich in gewissen Grenzen proportional zu der Kreatininkonzentration verhält. Der Nachteil dieses einfachen Verfahrens liẹt in seiner geringen Spezifität, da zahlreiche dem Kreatinin strukturell verwandte Körper, Ketone und Zucker ebenfalls zu einer Rotfärbung alkalischer Pikrinsäure führen können. Die methodologischen Probleme der Kreatininbestimmung nach JAFFÉ betrafen bisher vor allem die Entfernung oder quantitative Erfassung dieser interferierenden chromogenen Substanzen (vgl. 2), während die chemischen Vorgänge der Reaktion nur wenig bearbeitet wurden. Daher liegen weder über die Konstitution des Reaktionsproduktes noch über die Reaktionsgleichgewichte und deren Auswirkungen auf die Farbintensität bei Konzentrationsänderungen einzelner Partner eindeutige Ergebnisse vor. Von dem qualitativen Phänomen einer visuell beobachteten Farblöschung bei hohen Kreatininund $\mathrm{NaOH}-\mathrm{Konzentrationen}$ ausgehend, soll deshalb versucht werden, mit spektroskopischen und chromatographischen Methoden eine vorläufige Deutung der Reaktionsabläufe herbeizuführen.

\section{Methodik}

Spektroskopische Untersuchungen

Die spektroskopischen Untersuchungen wurden mit einem Spektralphotometer PMQ II (Zeiss) in Quarzküvetten von 0,5 und $2,0 \mathrm{~cm}$ Schichtdicke bei Wellenlängen zwischen 200 und $550 \mathrm{~nm}$ ausgeführt.

Untersucht wurden:

a) die Spektren von $12 \mathrm{~mm}$ bis $9,15 \mu \mathrm{M}$ Pikrinsäure in Tetrachlorkohlenstoff, 10 und $5 \mathrm{~N} \mathrm{HCl}$, in $\mathrm{H}_{2} \mathrm{O}$ und in 0,005 bis $6,4 \mathrm{~N} \mathrm{NaOH}$ Der Kreatiningehalt dieser Lösungen lag zwischen Null und $5 \mathrm{mg} / 100 \mathrm{ml}$ :

b) die Spektren zwischen 350 und $550 \mathrm{~nm}$ von PikrinsäureLösungen $(9,15 \mu \mathrm{M}$ bis $12 \mathrm{mM})$, denen bei konstanter $\mathrm{NaOH}-$ Konzentration (je $0,1-0,5 \mathrm{~N}$ ) steigende Mengen von Kreatinin ( $88 \mu \mathrm{M}$ bis $0,6 \mathrm{M}$ ) zugefügt wurden.

Untersuchungen zur Charakterisierung des Reaktionsproduktes

Darstellung der roten Kreatinin-Pikrinsäıre-Verbindung (nach 1. c. (3)) Einer heiß gesättigten Pikrinsäure-Lösung wurden mehrere Spatelspitzen Kreatinin zugegeben. Das ausgefallene Krcatininpikrat wurde mit $\mathrm{NaOH}$ in Lösung gebracht, bis ein tiefroter Farbton auftrat. Nach dem Erkalten wurde der in Eiswasser gekühlten Lösung schnell ein kleines Volumen $8 \mathrm{~N} \mathrm{HCl}$ zugefügt. Das ausgefällte rote Produkt wurde abzentrifugicrt und zur Entfernung überschüssiger Pikrinsäure mehrmals mit Äther extrahiert. Nach vollständiger Trocknung erfolgte die spektroskopische Untersuchung in 0,005 bis $2,0 \mathrm{~N} \mathrm{NaOH}$. 


\section{Dïnnscbichtchromatographische Untersucbungen}

Dünnschichtchromatographisch wurden untersucht: Pikrinsäure, Pikraminsäure, das rote Produkt und die Lösungen der JafféReaktion mit Kreatinin und Glucose. Die glucosehaltigen Lösungen waren bis zum Eintritt der Rotfärbung erhitzt worden. Die Chromatogramme wurden auf Kieselgelplatten (Kieselgel nach StaHL, Merck) von $0,25 \mathrm{~mm}$ Schichtdicke ausgeführt. Die Platten waren mit $0,1 \mathrm{M}$ Glykokoll auf $\mathrm{pH} 12,7$ gepuffert und luftgetrocknet. Vorversuche ergaben als geeignete Fließmittel: n-Butanol für die erste Fraktionierung und Äthanol/ $\mathrm{H}_{2} \mathrm{O}(60: 40 \mathrm{v} / \mathrm{v})$ für die weitere Auftrennung der noch am Startpunkt verbliebenen Fraktionen. Die $R_{F}$-Werte der ein- und zweidimensional entwickelten Chromatogramme wurden aus den Ergebnissen von je fünf Chromatogrammen berechnet. Alle Fraktionen wurden in je $2,0 \mathrm{~m} / 0,1 \mathrm{~N} \mathrm{NaOH}$ gelöst und spektroskopisch untersucht.

\section{Ergebnisse}

Einfluß der $\mathrm{OH}^{-}$-Ionenkonzentration auf das Spektrum der Pikrinsäure

Das Spektrum der Pikrinsäure zeigt in apolaren Lösungsmitteln (Tetrachlorkohlenstoff) eine schmale Bande bei $335 \mathrm{~nm}$ (Abb. 1). Beim Übergang zu stark sauren polaren Lösungsmitteln entwickelt sich eine Schulter im langwelligen Teil der $335 \mathrm{~nm}$-Bande. Mit zunehmender Möglichkeit zur Dissoziation der Pikrinsäure findet eine bathochrome Verschiebung des Absorptionsmaximums nach $357 \mathrm{~nm}$ statt. Eine zweite bathochrome Verschiebung erfolgt beim Übergang von wäßrigen zu alkalischen Pikrinsäure-Lösungen. Das Absorptionsmaximum liegt jetzt bei $388 \mathrm{~nm}$. Diese alkalitätsbedingte Bathochromie wird von einer der $\mathrm{OH}$-Ionenkonzentration proportionalen Steigerung der Absorptionsintensitäten begleitet.

Besondere Beachtung verdienen die alkalitätsbedingten Änderungen der Absorptionsintensitäten im langwelligen Bereich zwischen 450 und $550 \mathrm{~nm}$, in dem die Farbintensitäten der Jaffé-Lösung bei der Kreatininbestimmung gemessen werden. Während Pikrinsäure in wäßrigen Lösungen zwischen 450 und $550 \mathrm{~nm}$ nur sehr geringe Absorption zeigt, steigen sie in alkalischen Lösungen proportional zur $\mathrm{OH}^{-}$-Ionenkonzentration an. Unabhängig von der Pikrinsäure-Konzentration er-

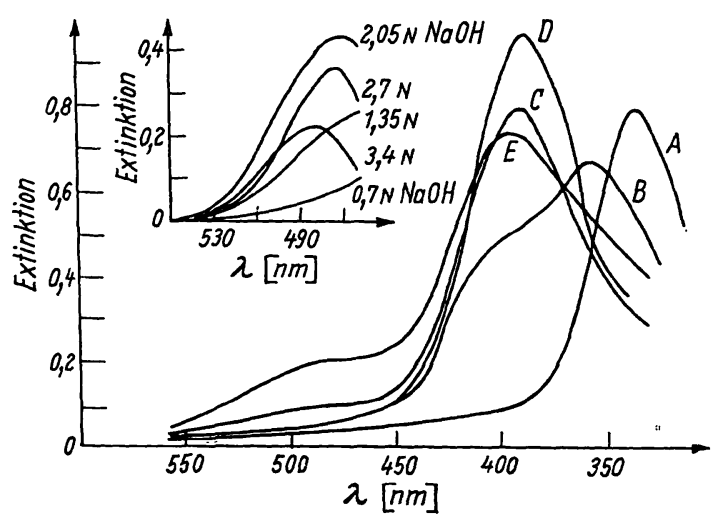

Abb. 1

Spektren der Pikrinsäure in $\mathrm{CCl}_{4}$ (A), in $\mathrm{H}_{2} \mathrm{O}(9,15 \mu \mathrm{M}, \mathrm{B})$, in $2 \mathrm{~N} \mathrm{NaOH}$ (C) und in $4 \mathrm{~N} \mathrm{NaOH}$ (D). E zeigt das Spektrum einer alkalischen Kreatinin-Pikrinsäure-Lösung. (88 $\mu \mathrm{M}$ Kreatinin, $0,5 \mathrm{~N} \mathrm{NaOH})$. In der bei Ânderungen der NaOH-Konzentration dargestellt (3,6 mM Pikrinsäure) reichen sie bei $3,5 \mathrm{~N} \mathrm{NaOH}$ die größten Werte, fallen aber bei weiterer Zunahme der Alkalität wieder auf die Ausgangswerte ab (Abb. 1). Den visuellen Ausdruck dieses Geschehens stellt die zú' und abnehmende Rotfärbung einer steigend alkalisierten Pikrinsäure-Lösung dar, deren relatives Absorptionsmaximum, gemessen gegen einen Leerwert wäßriger Pikrinsäure, bei 470 $490 \mathrm{~nm}$ liegt. Mit zunehmender $\mathrm{OH}^{-}$-Ionenkonzentration tritt eine langwellige Verschiebung des relativen Maximums mit Senkung der Absorptionsintensitäten ein.

Einfluß der $O H^{-}$-Ionenkonzentration auf das Spektrum der Pikrinsäure-Kreatininlösungen bei geringem Kreatiningehalt $(<10 \mathrm{mg} / 100 \mathrm{ml}$ )

Nach Ablauf der Jaffé-Reaktion mit Kreatinin bleiben die beschriebenen Grundformen der Pikrinsäure-Spektren bestehen. Die Absorptionsmaxima liegen hier bei $\mathrm{NaOH}-$ Konzentrationen unter $0,05 \mathrm{~N}$ bei $357 \mathrm{~nm}$, bei höheren Konzentrationen findet sich eine der früheren $388 \mathrm{~nm}$-Bande entsprechende bei $400 \mathrm{~nm}$. Ungeachtet der kräftigen Rotfärbung der Lösung erscheint die Form des Spektrums nur geringfügig verändert. Neben der Senkung der Absorptionsintensität kommt es zu einer kleinen bathochromen Verschiebung des Maximums. Im langwelligen Teil des Spektrums (zwischen 450 und $550 \mathrm{~nm}$ ) treten dagegen schon bei geringen Alkalikonzentrationen $(<0,1 \mathrm{~N})$ starke Erhöhungen der Absorptionsintensitäten auf, die gemessen gegen einen Leerwert gleich stark alkalischer Pikrinsäure ohne Kreatinin zwischen 470 und $490 \mathrm{~nm}$ wieder ein relatives Maximum ergeben. Eine Erhöhung der Alkalität führt $z u$ einer vorübergehenden Löschung der kreatinininduzierten Absorption (Abb. 2), der dann der eingangs beschriebene alkalibedingte Absorptionsanstieg folgt. Mit Pikrinsäure und hohen $\mathrm{NaOH}-K o n z e n t r a t i o n e n$ sowie mit Pikrinsäure, Kreatinin und geringen $\mathrm{NaOH}$ Konzentrationen kann eine visuell und spektroskopisch nicht unterscheidbare Rotfärbung der Lösung erhalten werden.

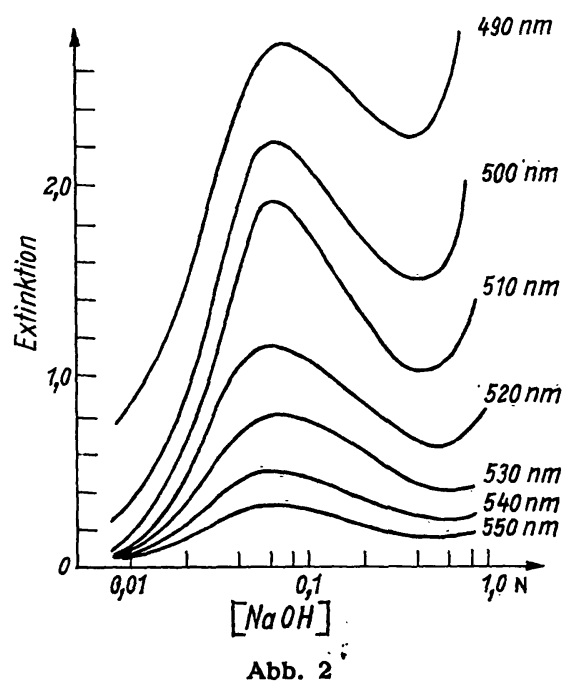

Abhängigkeit der Farbintensität von der $\mathrm{NaOH}-K o n z e n t r a t i o n ~ b e l$ Wellenlängen von $550-490 \mathrm{~nm}$.

1,3 $\mathrm{mm}$ Pikrinsäure, $36 \mathrm{~mm}$ Kreatinin, $\mathrm{d}=2 \mathrm{~cm}$ 
Einflußs der Kreatininkonzentration auf das PikrinsäureSpektrum bei konstanten $\mathrm{NaOH}$-Konzentrationen $(0,1-0,5 \mathrm{~N})$ Eine Proportionalität von Kreatininkonzentration und Absorptionsintensität im relativen Maximum (490 nm) besteht nur bis zu $10 \mathrm{mg} / 100 \mathrm{ml}$ Kreatinin. Das Maximum der Farbentwicklung wird aber ungeachtet der Linearität der Eichkurve erst dann erreicht, wenn die Kreatininkonzentration die Pikrinsäure-Konzentration überschreitet. Bei noch höheren Kreatininkonzentrationen kommt es nach anfänglicher Rotfärbung der Lösung innerhalb weniger Minuten zu einer Farblöschung. Das Spektrum einer solchen Lösung besitzt. bei $388 \mathrm{~nm}$ ein Absorptionsmaximum und gleicht dem einer stark alkalischen Pikrinsäure-Lösung. Zugabe von Pikrinsäure läßt die Rotfärbung wieder eintreten. Gleiches kann, wenn auch in minderem Maße, durch Zugabe von Mineralsäuren erreicht werden. Eine Senkung der $\mathrm{NaOH}-K o n z e n t r a t i o n$ dieser "kreatininübersättigten“ Lösungen verhindert oder verzögert die Farblöschung.

Spektroskopisches Verbalten der isolierten roten Substanz in neutralen und alkalischen Lösungen

Die isolierte rote Substanz löst sich schlecht in Wasser, gut dagegen in alkalischen Lösungsmitteln. Ihr Spektrum entspricht dem einer wäßrigen Pikrinsäure-Lösung mit Absorptionsmaximum bei $357 \mathrm{~nm}$. Ganz anders verhält sich das Spektrum dieser Substanz in leicht alkalischen Lösungen $(0,05-0,1 \mathrm{~N} \mathrm{NaOH})$. Im langwelligen Bereich zeigen sich die der Jaffé-Lösung entsprechenden hohen Extinktionen (Abb. 3). Es bildet sich sogar ein zweites, wenn auch nur schwach angedeutetes

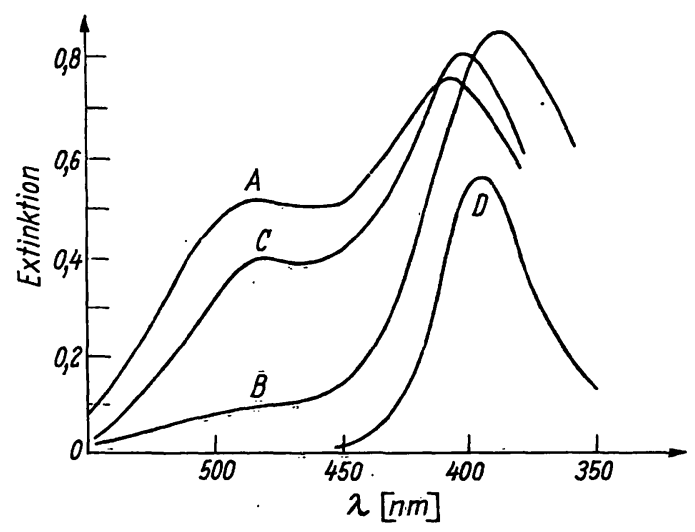

Abb. 3

Spektren der isolierten Kreatinin-Pikrinsäure-Verbindung in $0,05 \mathrm{~N}$ $\mathrm{NaOH}$ (A), in 2N NaOH (B) und der chromatographisch getrennten der Fraktionen $\mathrm{C}_{1} \mathrm{I}_{3}$ bzw. $\mathrm{BII}_{3}$ in $0,5 \mathrm{~N} \mathrm{NaOH}$ absolutes Maximum zwischen 470 und $490 \mathrm{~nm}$ aus. Das erste Maximum liegt abweichend von der Jaffé-Lösung $(395-400 \mathrm{~nm}$ ) bei $410 \mathrm{~nm}$. Eine Erhöhung der $\mathrm{NaOH}-$ Konzentration führt wieder zu einer Depression der Extinktionen im langwelligen Bereich und zu einer Verschiebung des Maximums nach $388 \mathrm{~nm}$.

Dünnscbicbtcbromatographische Ergebnisse (vgl. Abb. 4 und Tab. 1)

Die chromatographische Fraktionierung und spektroskopische Untersuchung ergaben eine völlige Identität zwischen Jaffé-Lösung mit Kreatinin und der isolierten roten Verbindung. Die immer wieder als Reaktionsprodukt angegebene Pikraminsäure zeigte bei der Fraktionierung mit Butanol ein von der Jaffé-Lösung abweichendes Verhalten $\left(R_{F} 0,26, v g l\right.$. Tab. 1), während bei der Jaffé-Reaktion mit Glucose unter anderem offensichtlich Pikraminsäure entsteht (Fraktion AI 2 mit $R_{F} 0,26$ ). Die erste Fraktionierung der Jaffé-Lösung mit Kreatinin und der roten Verbindung mit Butanol ergab lediglich eine Abtrennung der Pikrinsäure $\left(R_{F}\right.$ $0,85 ; 0,86)$. Die am Startpunkt verbliebenen Flecken zeigten mit Äthanol- $\mathrm{H}_{2} \mathrm{O}$ ein völlig konkordantes Verhalten. Die schnellste Fraktion bestand noch einmal aus Pikrinsäure (BII1/ $R_{F} 0,82 ; \mathrm{CII} 1 / R_{\text {F }} 0,81$ ).

Die zweite Fraktion (BII2 und CII2, $R_{F} 0,75$ ) erwies sich spektroskopisch als eigentliche farbgebende Kom-

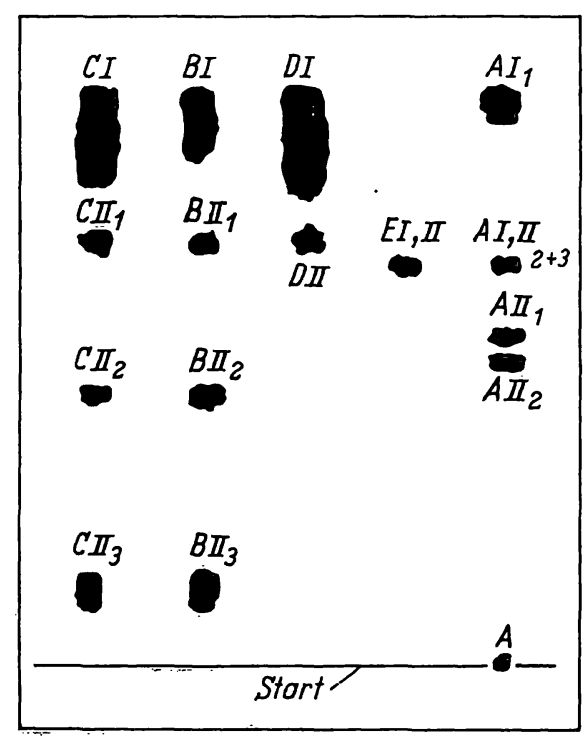

Abb. 4

Eindimensionales Chromatogramm mit n-Butanol und Äthanol/ $\mathrm{H}_{2} \mathrm{O}$ entwickelt. Bezeichnung und $R F$-Werte vgl. Text

Tab. 1

\begin{tabular}{|c|c|c|c|c|c|c|c|c|c|c|}
\hline \multirow{2}{*}{$\begin{array}{l}\text { Erste Fraktionierung } \\
\text { mit n-Butanol }\end{array}$} & \multirow{2}{*}{ 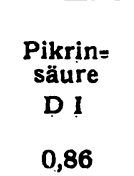 } & \multirow{2}{*}{$\begin{array}{l}\text { Pikramin- } \\
\text { säure } \\
\text { E I } \\
0,26\end{array}$} & \multicolumn{4}{|c|}{$\begin{array}{l}R_{\mathbf{F}} \text {-Werte } \\
\text { isolierte } \\
\text { Rote Substanz }\end{array}$} & \multicolumn{4}{|c|}{$\begin{array}{l}\text { Jaffé-Lösung mit Glucose } \\
\text { nach ERhitzen }\end{array}$} \\
\hline & & & & $\begin{array}{l}\text { Rest am } \\
\text { Startpunkt }\end{array}$ & $\begin{array}{l}C 1 \\
0.86\end{array}$ & $\begin{array}{l}\text { Rest am } \\
\text { Startpunkt }\end{array}$ & $\mathrm{Al}_{2}$ & $\mathrm{AI}_{2}$ & & $\begin{array}{l}\text { Rest am } \\
\text { Startpunkt }\end{array}$ \\
\hline $\begin{array}{l}\text { Zweite Fraktionierung } \\
\text { der am Startpunkt } \\
\text { verblieberien Flecken } \\
\text { mit Athariol/H, } \mathrm{H}_{2} \mathrm{O} \\
(60: 40 \mathrm{v} / \mathrm{v})(60-40)\end{array}$ & $\begin{array}{c}\text { Kontrolle } \\
0,81\end{array}$ & $\underset{0,8}{\text { Kontrolle }}$ & & $\begin{array}{l}\mathrm{BII}_{1} \\
0,82 \\
\mathrm{BII}_{2} \\
0,75 \\
\mathrm{BII}_{2} \\
0,22\end{array}$ & & $\begin{array}{l}\mathrm{ClI}_{2} \\
0,81 \\
\mathrm{CII}_{2} \\
0,75 \\
\mathrm{CII}_{2} \\
0,23\end{array}$ & & & & $\cdot$ \\
\hline
\end{tabular}


ponente (Abb. 3). Bei BII3 handelt es sich um eine gelblich gefärbte Substanz, die spektroskopisch eine schmale Bande mit Maximum bei $410 \mathrm{~nm}$ zeigte (Abb. 3). $\mathrm{Ob}$ es sich hierbei um einen Artefakt oder um eine bei der Reaktion entstehende Verbindung handelt, kann noch nicht entschieden werden.

\section{Diskussion}

Auf Grund der vorliegenden Ergebnisse können wir als Ursache der Rotfärbung einer alkalischen PikrinsäureLösung durch Kreatinin die Entstehung einer roten Verbindung aus Kreatinin und Pikrinsäure annehmen. Diese Verbindung aus je einem Molekül Pikrinsäure und Kreatinin, die schon früher als Reaktionsprodukt angesehen wurde (4-7), zeigte sowohl spektroskopisch als auch chromatographisch ein mit der Jaffé-Lösung identisches Verhalten. Wir müssen daher die oft für die Rotfärbung verantwortlich gemachte Reduktion der Pikrinsäure zu Pikraminsäure (8-10) ablehnen.

$\mathrm{Da}$ die Rotfärbung alkalischer Pikrinsäure nicht nur mit Kreatinin, sondern auch mit aliphatischen und zyklischen Carbonylverbindungen, Säureamiden und Imiden, Estern, Äthylenverbindungen, Cyan-, Nitround Diazokörpern erzielt werden kann, versuchten Weisse und Tropp (11) eine einheitliche Deutung der Reaktion aller in der Kälte jaffépositiver Substanzen über eine diesen Molekülen eigene aktive Methylenund Methingruppe zu geben. Die Jaffé-Reaktion mit Kreatinin stellt somit den Spezialfall einer allgemeinen Reaktion zwischen nitrosubstituierten Benzolen und Molekülen mit aktiver Methylengruppe dar. Allein aus der visuell beobachteten Rotfärbung kann aber nicht auf eine allgemeine Reaktion geschlossen werden.

Im Falle des Kreatinins tritt nur dann eine Rotfärbung auf, wenn das Molekül eine ungehinderte Fähigkeit zur Keto-Enol-Tautomerie besitzt (5). Die auf Grund dieses Ergebnisses entwickelte Vorstellung einer toten Salzverbindung aus Pikrinsäure und basischem KreatininEnol kann aber nicht zutreffen, da nichtbasische Körper wie Aceton nach unseren spektroskopischen Untersuchungen sich dem Kreatinin gleich verhalten und da das schwach basische Kreatinin $\left(K_{B}=3,7 \cdot 10^{-11}\right)$ in dem stark alkalischen Medium basische Eigenschaften nicht mehr besitzen kann. Stellen wir uns die KetoEnol-Tautomerie als das Gleichgewicht zwischen zwei Säuren und ihrem gemeinsamen Anion vor, so wird Kreatinin ganz besonders in alkalischer Lösung unter Abspaltung eines Protons Eigenschaften eines Säurerestes annehmen (Abb. 5).

Der eigentliche Grund der Rotfärbung wird aber bei der Pikrinsäure zu suchen sein, kann doch durch genügende Alkalisierung eine von Kreatinin nicht zu übertreffende Rotfärbung erzielt werden. Diese alkalibedingte Bathochromie der Pikrinsäure beruht auf einer Erweiterung der lichtabsorbierenden Systeme mit mesomerer Stabilisierung der Grundzustände und Senkung der Anregungsenergie, die sich formal durch die Aufrichtung des doppelt gebundenen O-Atoms der Nitro-
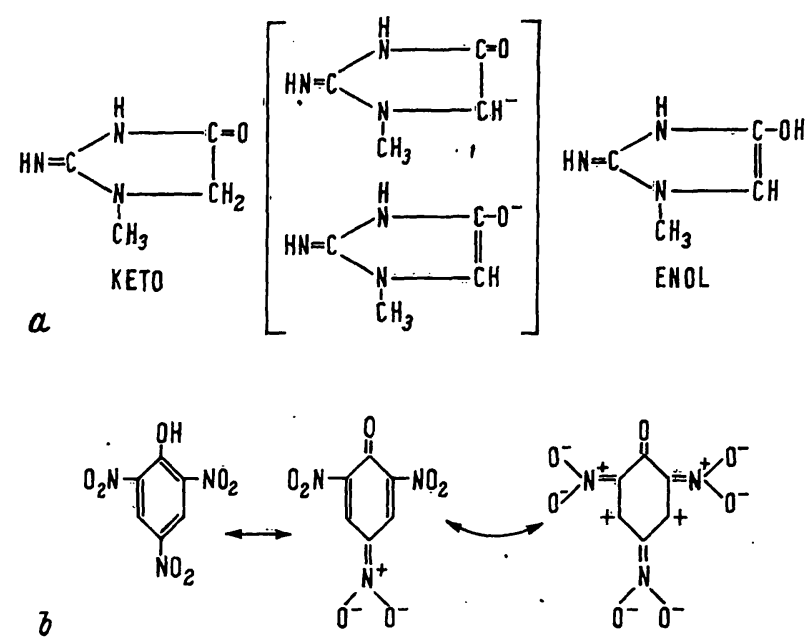

Abb. 5

a) Keto-Enol-Tautomerie des Kreatinins als Gleichgewicht zwischen zwei Säuren und ihrem gemeinsamen Anion b) Formale Darstellung prototrop tautomerer und mesomerer Er-

gruppe erklären läßt (Abb. 5). Begünstigt werden diese Vorgänge bei der Pikrinsäure durch den starken negativ mesomeren Effekt ihrer Nitrogruppen, der zu einem Elektronenabzug aus dem Ring und dadurch zu dessen Positivierung führt.

Die für den Reaktionseintritt notwendige Alkalität kann einmal zu einer Enolisierung des Kreatinins, andererseits zu einer Positivierung. des N-Atoms an der Pikrinsäure führen. Wahrscheinlich werden hier die Pole liegen, die zu der Bindung beider Partner beitragen. Genaue Aufschlüsse über die Art der Verbindung und die beteiligten Gruppen können aber nur eingehende Analysen der IR-Spektren geben.

Die Jaffé-Reaktion stellt nach allen bisher erhobenen Befunden eine Gleichgewichtsreaktion dar, die formal durch

$$
\frac{[\text { Pikrinsäure-Kreatinin] }}{\text { [Kreatinin] [Pikrinsäure }]}=\mathrm{K}
$$

dargestellt werden kann (12). Auf Grund unserer Ergebnisse bei extremer Variation der Parameter müssen wir jedoch einen noch komplizierteren Reaktionsmechanismus annehmen. $\mathrm{Da}$ das Maximum der Farbintensität erst dann erreicht wird, wenn der Kreatiningehalt der Lösung die Pikrinsäure-Konzentration überschreitet, kann sich nicht alles vorhandene Kreatinin an der Reaktion beteiligt haben, was durch die Annahme eines Gleichgewichtes zwischen reagibler Enol- und inerter Ketoform des Kreatinins zu erklären ist. Die Lage dieses Gleichgewichtes wird im wesentlichen von der Konzentration der Natronlauge beeinflußt. Eine Erhöhung derselben führt aber nicht zu der erwarteten Steigerung der Farbintensität, sondern zur Farblöschung. Ein weiterer Reaktionsmechanișmus muß daher das Geschehen komplizieren.

Auf Grund einer wenig bekannten Tatsache lassen sich hierüber gewisse Schlüsse ziehên. Die Pikrinsäure ist mit drei Nitrogruppen substituiert, an die sie nach ihrer Aufrichtung in alkalischem Milieu je ein Kreatinin- 
molekül anlagern kann. Es besteht daher die Möglichkeit einer Bildung von Di-Kreatinin-Pikrinsäure bei einem Überschuß von $\mathrm{NaOH}$ oder Kreatinin. Tatsächlich konnte aus einer Lösung mit einer gegenüber der Pikrinsäure-Konzentration hohen Kreatinin-und $\mathrm{NaOH}$ Konzentration eine Verbindung aus einem Molekül Pikrinsäure und zwei Molekülen Kreatinin isoliert werden (6), die in alkalischer Lösung keine Rotfärbung aufwies. Neben dem Keto-Enol-Gleichgewicht kann bei der Jaffé-Reaktion noch ein Gleichgewicht zwischen Kreatinin-Pikrinsäure und Di-Kreatinin-Pikrinsäure bestehen, das sich bei einer Erhöhung der Kreatinin- oder $\mathrm{NaOH}-$ Konzentration zugunsten der Di-KreatininPikrinsäure-Bildung verschiebt.

\section{Schlußbetrachtung}

Ungeachtet der noch offenen Probleme können wir aus den vorliegenden spektroskopischen und chromatographischen Untersuchungen der Jaffé-Reaktion mit Kreatinin folgende Schlüsse ziehen:

1. Das für die Rotfärbung der Lösung verantwortliche Reaktionsprodukt ist sicher keine Pikraminsäure. Es handelt sich um ein Assoziat aus Pikrinsäure und Kreatinin, das keine reine kovalente Bindung besitzt und nur in alkalischen Lösungen stabil ist.
2. Die Reaktion stellt eine mehrschichtige Gleichgewichtsreaktion dar, die unter Umständen zur Bildung weiterer Pikrinsäure-Kreatininassoziate mit anderem Farbverhalten führt.

3. $\mathrm{Zu}$ hohe $\mathrm{NaOH}$ - und Kreatininkonzentrationen müssen bei der Jaffé-Reaktion vermieden werden. Maximale Extinktionen werden bei $\mathrm{NaOH}-\mathrm{Konzentration}$ von $0,05-0,06 \mathrm{~N}$ erhalten (Abb. 3). Bei vielen Methoden liegen sie aber um $0,2 \mathrm{~N} \mathrm{NaOH}$. $\mathrm{Da}$ die kreatinininduzierte Farblöschung bei hohen $\mathrm{NaOH}-\mathrm{Konzentra-}$ tionen schneller eintritt, sollte diese auch wegen des sehr kleinen proportionalen Verhältnisses von Extinktion zur Kreatininkonzentration niedergehalten werden.

4. Die Linearität der Eichkurve bei $490 \mathrm{~nm}$ kann nur bis zu 8 , höchstens $10 \mathrm{mg} / 100 \mathrm{~m} /$ Kreatinin gewährleistet werden. Im langwelligen Teil (bis zu $550 \mathrm{~nm}$ ) steigt sie bis zu 100 und $\mathrm{mehr} \mathrm{mg} / 100 \mathrm{ml}$ an. Eine Messung außerhalb des relativen Maximums (490 nm) sollte daher vorgezogen werden.

5. Unterschiede in der $\mathrm{NaOH}-$ Konzentration zwischen Leerwert, Standard und Probe sind auf jeden Fall zu vermeiden. Die Standards sollten eiweißhaltig sein und wie Serum bebandelt werden, sofern die gebräuchlichen Methoden zur Kreatininbestimmung herangezogen werden.

\section{Literatur}

1. JAFFÉ, M., Hoppe-Seyler's Z. physiol. Chem. 10, 391 (1886). 2. Dubach, U. C., I. Metz und P. Schmid, Klin. Wschr. 45, 621 (1967). - 3. GreENwald, I., J. biol. Chemistry 80, 104 (1928). 4. Reissert, A., Chem. Bet. 37, 831 (1904). - 5. Greenwald, I., J. Amer. chem. Soc. 47, 1440 (1925). - 6. Greenwald, I., J. biol. Chemistry 77, 539 (1928). - 7. AnsLov, W. K. und H. King, J. Chem. Soc. London 1210 (1929). - 8. Chapman,
Analyst 34, 475 (1909). - 9. Dox, A. W., Hoppe-Seyler's Z. physiol. Chem. (1925). - 10. Pilsum, J. F. van, R. P. Martin, E. Krro und J. Hess, J. biol. Chemistry 222, 225 (1956). - 11. Wersse, W. und C. Tropp, Hoppe-Seyler's Z. physiol. Chem. 178, 125 (1928). - 12 Dunicz, B. L., Clin. chim. Acta Amsterdam 9, 203 (1964).

Dr. H. P. Seelig

1 Berlin 19

Patholog. Institut der Freien Universität im Klinikum Westend 